\title{
La formation professionnelle continue, une catégorie de la négociation interprofessionnelle encore pertinente?
}

Créée dans la dynamique du programme du Conseil national de la résistance, la commission Langevin-Wallon affirme, dans son rapport remis en 1947, « la possibilité pour tous de poursuivre au-delà de l'école et durant toute leur existence le développement de leur culture intellectuelle, esthétique et professionnelle, civique et morale ». Elle dessine ainsi une vision de la formation du citoyen dans laquelle le professionnel n'est qu'un élément marginal dans une conception centrée sur l'épanouissement personnel. La loi du 16 juillet 1971, acte initiateur du système de formation professionnelle continue, place d'emblée celle-ci sous la double référence de la sphère du travail et de la sphère éducative et culturelle ${ }^{2}$. Le code du travail actuellement en vigueur fait de la formation professionnelle continue un élément de la formation professionnelle tout au long de la vie ${ }^{3}$ : il la pose en « obligation nationale » que « l'Etat les collectivités locales, les établissements publics et privés, les associations, les organisations professionnelles syndicales et familiales ainsi que les entreprises » (article D. 6312-1) doivent concourir à assurer. Dans ce cadre, il définit la formation professionnelle continue comme ayant pour objet « de favoriser l'insertion ou la réinsertion professionnelle des travailleurs, de permettre leur maintien dans l'emploi,

1. Sociologue, CNRS-IRISSO - Université Paris-Dauphine.

2. Le préambule de la loi de 1971 précise : " La formation professionnelle continue fait partie de l'éducation permanente. Elle a pour objet de permettre l'adaptation des travailleurs au changement des techniques et des conditions de travail, de favoriser leur promotion sociale par l'accès aux différents niveaux de la culture et de la qualification professionnelle et leur contribution au développement culturel, économique et social. »

3. Celle-ci est entendue comme " une formation initiale, comprenant notamment l'apprentissage et des formations ultérieures, qui constituent la formation professionnelle continue, destinés aux adultes et aux jeunes déjà engagés dans la vie professionnelle » (article L. 6111-1). 
de favoriser le développement de leurs compétences et l'accès aux différents niveaux de la qualification professionnelle, de contribuer au développement économique et culturel et à leur promotion sociale » (article L. 6311-1). La vision sociétale de la formation professionnelle continue s'est donc considérablement transformée au cours des soixante dernières années. Dès lors, deux questions fortement articulées se posent : quels sont les facteurs structurants de ces transformations? Comment la négociation collective interprofessionnelle ${ }^{4}$ dans ce domaine les a-t-elle pris en compte?

Un consensus s'est formé depuis plusieurs années parmi les analystes des relations professionnelles pour contester la vision de Dunlop (1958) de règles façonnées par les évolutions des contextes économiques et organisationnels (Reynaud et al., 1990), et mettre en avant l'autonomie des acteurs des relations professionnelles dans les modes d'appropriation de ces contraintes qu'ils élaborent et dans les compromis qu'ils construisent dans la négociation à travers des processus de régulation conjointe (Reynaud, 1989). Pour autant, la question de la caractérisation de ces modes d'appropriation reste ouverte. Autrement dit, quels liens peut-on distinguer entre les évolutions économiques et les politiques de gestion de la main-d'œuvre qui leurs sont liés, les enjeux qui émergent dans la négociation et les règles de contenu et de gouvernance élaborées par les acteurs du système de négociation de la formation professionnelle continue (FPC) ? L'autre question qui se pose est celle du rythme et du contenu des évolutions. Celles-ci s'effectuent-elles selon des ruptures, des changements structurels propres à la configuration sociétale française (Silvestre, $19866^{5}$ ) ou au contraire à travers des processus de dépendance de sentier ${ }^{6}$ dans lesquels on peut observer des éléments d'hybridation entre l'ancien et le nouveau, permettant aux systèmes de régulation de s'adapter aux changements ?

Dans cet article, on tentera de répondre à ces questions en analysant conjointement les évolutions des contextes économiques, les transformations des enjeux et du système d'acteurs qui ont conduit aux accords marquants de la période 1970-2003 (I), puis en s'interrogeant sur les dynamiques propres à l'accord de janvier $2009^{7}$ (II).

4. Dans cet article, on se centrera sur les dispositifs issus de la négociation collective ou des lois qui en ont généralisé les dispositions. Notre analyse prend donc l'accord de juillet 1970 comme date d'origine et ne traitera pas des lois sur la formation professionnelle des adultes qui l'ont précédé. Pour une histoire de la formation professionnelle, on se reportera aux travaux de Tanguy (1999, 2001), Dubar (1999, 2004), Santelman (2001).

5. Dans la référence citée, Silvestre oppose la plasticité des règles propres au modèle allemand aux changements structurels qui caractérisent le modèle français.

6 . Les analyses issues de l'économie et des sciences politiques insistent sur ce processus par lequel les acteurs se tournent plutôt vers des choix assurant une certaine continuité (David, 1985 ; Pierson, 1995).

7. L'analyse de la négociation de l'accord de janvier 2009 et du système d'acteurs dans lequel elle est insérée repose sur un travail d'enquête réalisé avec Carole Tuchszirer (CEE) dans le cadre de la convention de l'IRES avec la DARES Les organisations patronales. Continuités et mutations des formes de représentation du patronat (Pernot, Vincent, 2011). L'exploitation que nous avons réalisée de cette enquête constitue le chapitre 4 de ce rapport. Pour mener à bien ce travail, nous avons effectué des entretiens avec des représentants de toutes les organisations patronales ainsi qu'avec des représentants d'organisations syndicales parties prenantes des négociations de 2009 et parfois de 2003. 


\section{La construction d'un système par sédimentation (1970-2003)}

La plupart des normes qui régissent le domaine de la formation professionnelle continue depuis 1970 résultent d'une articulation entre accord interprofessionnel et loi, dans laquelle la loi reprend plus ou moins complètement les dispositions négociées ; on parle donc souvent de dispositifs pour évoquer ces ensembles de règles. Quelques dates, signalées comme autant de ruptures marquantes, reviennent dans l'histoire de la construction de ces règles depuis 40 ans : 1970-1971, 1983-1984, 1991 et 2003-2004. Pourtant, en particulier dans la dernière période, des éléments centraux existent déjà en pointillé dans les dispositifs antérieurs. On peut donc s'interroger sur l'origine de ces points d'inflexion, sinon de rupture. Le contexte économique, et son influence sur les politiques de gestion de la main-d'œuvre des entreprises, ne peut manquer de peser sur les négociations, même s'il est médiatisé par les stratégies des acteurs dans la construction des compromis. A cet égard, la remarque de Comaille (2001:35) à propos des lois sur la formation professionnelle continue entre 1959 et 1993 - [elles] « apparaissent au moment de leur adoption comme une consécration légale d'une nécessité économique, sociale et culturelle »- peut être transposée aux accords interprofessionnels de cette période. Ces nécessités constituent autant de composantes des référentiels de politique publique que les acteurs sociaux parties prenantes de nombre d'instances d'expertise (Commissariat général du plan, groupe de contrôle de la formation professionnelle, groupe de politique contractuelle...) ont contribué à construire. Dans cette partie sont analysés, sur la période 1970-2003, ces enchaînements entre conjoncture économique, émergence des enjeux, dynamiques du système d'acteurs de la négociation collective sur la FPC et formes de traduction des enjeux concrétisées dans les accords.

\section{I.1. Les enjeux : de l'emploi à l'individualisation}

La loi de 1971 est porteuse d'une tension entre une finalité économique - la formation professionnelle continue - et une finalité sociale de la formation - la formation permanente -, dans laquelle la première devient rapidement dominante avec l'irruption de la crise économique au milieu des années 1970. Si, dans une première période, cette finalité économique s'organise suivant des enjeux d'abord liés à l'emploi, dès les années 1990, ceux liés à une vision de l'amélioration de l'employabilité ${ }^{8}$ des salariés afin de faciliter la construction de parcours professionnels s'affirment. Dans cette optique, l'accord de 2003 apparaît plus comme l'aboutissement d'une

8. La conférence internationale du travail ( $88^{\mathrm{e}}$ session) a ainsi défini la notion d'employabilité en juin 2000 : « les compétences, connaissances, qualifications qui renforcent les aptitudes des travailleurs à trouver, conserver un emploi, progresser au niveau professionnel, s'adapter au changement, trouver un autre emploi s'ils le souhaitent ou s'ils ont été licenciés et s'intégrer plus facilement au marché du travail dans les différentes périodes de leur vie » (cité dans CGP, 2003:6). 
logique qui prend ses racines au début de la décennie précédente que comme un accord totalement novateur.

\section{De l'accompagnement des reconversions à la priorité à l'insertion des jeunes (années 1970-1980)}

Le contexte économique fortement contradictoire des années 1960 marque les stratégies des acteurs. Tandis que la croissance économique favorise le fonctionnement des marchés internes et une vision de la formation (entendue comme formation permanente) comme outil d'épanouissement personnel et de promotion sociale, l'accentuation de la concurrence consécutive à l'ouverture des marchés conduit à des restructurations qui donnent lieu à une hausse significative des conflits sociaux. La création du Fonds national de l'emploi (FNE) est à cet égard significative d'une volonté publique d'intervenir en s'appuyant sur des dispositifs centrés sur la formation pour diminuer le coût social de ces restructurations et peser sur un chômage considéré alors par les pouvoirs publics et le patronat comme conjoncturel. Néanmoins, l'activité revendicative sur l'emploi s'accroît et les organisations syndicales demandent dès 1967, avec peu de succès, l'ouverture de négociations sur les salaires, le droit syndical et l'emploi. Le constat de Grenelle, qui fait suite aux mouvements de mai 1968, enjoint les organisations professionnelles et syndicales à négocier dans un court délai sur la sécurité de l'emploi. Il prend acte de la nécessité d'« étudier les moyens permettant d'assurer avec le concours de l'Etat, la formation et le perfectionnement professionnel ». L'accord de 1969 sur la sécurité de l'emploi traitait déjà indirectement de la formation : en témoigne la création dans chaque profession de commissions paritaires de l'emploi dont les attributions de suivi de la situation de l'emploi concernaient également l'étude des moyens de formation et la formulation de propositions en la matière. L'accord interprofessionnel de juillet 1970 se place donc dans la dynamique de cet accord en ce qui concerne la prévention des licenciements et les moyens mis en œuvre pour adapter les salariés aux transformations de l'appareil productif et de l'organisation du travail. Le «traumatisme» du mouvement de mai 1968 et cette vision d'un chômage d'adaptation dans un contexte de croissance propre à l'environnement des Trente Glorieuses expliquent l'acceptation par le CNPF du principe d'une contribution des entreprises pour financer la formation. Dans cette période où se mettent en place les prémisses du système paritaire, on peut considérer que s'échangent la liberté de disposition par les entreprises de cette contribution contre une élaboration négociée des règles de fonctionnement de ces dispositifs (d'Iribarne, Lemaître, 1987). L'entrée de la FPC dans le code du travail par le dispositif de 1970-1971 se place dans cette logique alors que la formalisation du congé individuel de formation (CIF) traduit la persistance de celle d'éducation permanente (encadré 1). 


\section{Les dispositifs 1970-2003}

L'accord national interprofessionnel du 9 juillet 1970 :

- définit un droit de consultation du comité d'entreprise dans le cadre de l'élaboration du plan de formation ;

- élargit les prérogatives des commissions paritaires nationales de l'emploi (CPNE) (créées par l'accord sur la sécurité de l'emploi de 1969) à la formation professionnelle.

La loi du 16 juillet 1971 :

- introduit, pour les entreprises de plus de dix salariés, la contribution obligatoire sur les salaires ;

- définit le congé individuel de formation (le principe avait été introduit dans la loi de 1966);

- institue la possibilité d'institution paritaire de gestion des fonds de formation : les Fonds d'assurance formation (FAF).

L'accord interprofessionnel de 1983 :

- définit de nouvelles modalités d'insertion des jeunes par l'alternance (notamment le contrat de qualification);

- institue une contribution obligatoire des entreprises au financement de l'alternance ;

- prévoit la possibilité de mettre en place des organismes collecteurs de cette taxe.

La loi du 24 février 1984 (dite loi Rigout) introduit l'obligation de négocier sur les objectifs et les moyens de la formation professionnelle.

La loi du 4 juillet 1990 :

- définit le crédit formation individualisé (CFI) ;

- institue un droit individuel à la qualification en tant que principe général du code du travail.

L'accord interprofessionnel du 3 juillet 1991 (repris dans la loi du 31 décembre 1991) :

- consacre le droit à la formation et à la qualification ;

- définit un droit au bilan de compétences ;

- étend le congé individuel de formation ;

- institue le co-investissement pour les formations diplômantes ;

- étend les prérogatives des CPNE ;

- définit une obligation quinquennale de négocier sur la formation professionnelle et précise ses thèmes obligatoires ;

- étend l'obligation de financement aux entreprises de moins de dix salariés.

La loi du 20 décembre 1993 (dite loi quinquennale) :

- transfère aux régions les compétences en matière de formation professionnelle des jeunes ;

- pose les principes d'une réforme de la collecte.

L'avenant du 5 juillet 1994 à l'accord interprofessionnel de 1991 fait suite à la loi quinquennale. II crée les organismes paritaires collecteurs agrées (OPCA). 


\section{L'accord de 2003 :}

- crée un droit individuel à la formation (DIF) d'une durée de 20 heures par an, cumulables sur six ans, ouvert aux salariés en CDI ayant au moins un an d'ancienneté, et aux salariés en CDD présents depuis quatre mois dans l'entreprise ;

- met en place une période de professionnalisation pour certaines catégories de salariés (ceux dont la qualification est inadaptée à l'évolution des technologies et les salariés en deuxième partie de carrière), soit à l'initiative du salarié (DIF), soit à l'initiative de l'employeur (plan de formation), hors temps de travail sous un certain nombre de conditions ;

- fusionne les différents contrats de formation en alternance en un contrat de professionnalisation ouvert aux jeunes (moins de 26 ans) et aux adultes demandeurs d'emploi ;

- institue trois catégories d'actions de formation dans le plan de formation : d'adaptation au poste de travail (temps de travail effectif, rémunération maintenue), liées à l'évolution des emplois ou participant au maintien dans l'emploi (ne s'imputent pas sur le contingent d'heures supplémentaires dans la limite de 50 heures par an), et de développement des compétences (peuvent se dérouler en dehors du temps de travail);

- modifie et augmente la contribution des employeurs ;

- modifie la périodicité des négociations de branche, qui ont désormais lieu tous les trois ans au lieu de cinq. Par ailleurs, l'ANI liste une série de thèmes obligatoires, dont les priorités du DIF et des périodes et contrats de professionnalisation ;

- contraint à la mise en place d'observatoires prospectifs des métiers et des qualifications.

Au début des années 1980, dans un contexte de forte augmentation du chômage et de restructuration profonde de l'appareil productif, deux difficultés majeures apparaissent : l'insertion professionnelle des jeunes non qualifiés se fait plus ardue, tandis que les entreprises peinent à recruter du personnel qualifié dans de nombreux secteurs. Ce double mouvement réinterroge les modes d'accès à la qualification ainsi que les responsabilités respectives de l'Etat, des entreprises, des acteurs sociaux et des régions. Pour les pouvoirs publics, une meilleure qualification des jeunes, destinée à favoriser leur insertion, passe par l'augmentation et la diversification des voies d'accès au diplôme. Pour les entreprises, il est nécessaire de rapprocher l'offre de formation des différents niveaux où se définissent les besoins en qualification. La première option se concrétise dans l'ordonnance de mars 1982. Elle ouvre un droit à une formation sanctionnée par un diplôme et prévoit que les jeunes suivant une formation alternée peuvent obtenir leurs diplômes par unités capitalisables ou partiellement par validation des acquis. La seconde option se centre sur la reconnaissance d'autres voies d'accès que le diplôme à la qualification professionnelle, en ouvrant de 
nouvelles voies de certification dans lesquelles l'autorité compétente n'est plus l'Etat mais peut être une instance paritaire de la profession. Est ainsi mis en avant, pour parvenir à une plus grande implication des entreprises, le rôle des branches professionnelles. L'accord interprofessionnel de 1983 se place dans cette logique. Il définit plusieurs dispositifs d'insertion des jeunes, dont les contrats de qualification, pour lesquels « les commissions paritaires nationales de l'emploi pourront indiquer les qualifications professionnelles ou les préparations au diplôme de l'enseignement technologique qui leur paraissent devoir être développées ». Il institue une contribution obligatoire des entreprises au financement de l'alternance.

\section{La montée de l'individualisation (années 1990-2000)}

A l'aube des années 1990, la pérennité d'un taux de chômage élevé qui ne touche plus seulement les jeunes tend à ébranler les marchés internes et à remettre en cause les trajectoires professionnelles qui s'y étaient construites. Parallèlement, les restructurations du modèle productif et les transformations de l'organisation du travail dont elles sont porteuses ont des conséquences en termes de précarisation du contrat de travail et de transformation des modes de gestion de la main-d'œuvre avec le développement de la flexibilité. Ceux-ci prennent appui sur l'émergence d'un « modèle de la compétence » (Zarifian, 1988) fondé sur l'importance d'une stratégie de qualité articulée à une nouvelle organisation du travail et de nouvelles politiques de gestion de la main-d'œuvre. Ces dernières sont centrées sur la mobilisation des compétences individuelles pour faire face à des situations professionnelles multiples et sur la construction des compétences des salariés par la formation. Ce modèle tend à ébranler les modalités de régulation de la relation salariale, en y introduisant une plus grande individualisation dans laquelle la formation professionnelle apparait comme un enjeu central du maintien dans l'emploi et du déroulement de carrière. Les années 1990 correspondent à la période de montée du thème de la compétence dans le débat social (Cannac, 1985 ; d'Iribarne, 2001 ; Tallard, 2001). Le point d'orgue en est les assises de Deauville du MEDEF en 1998. Analysant les travaux de ces assises, Reynaud (2001) voit dans l'introduction de la notion de compétence l'émergence d'un nouveau type d'échange salarial performance/employabilité. Dans un tel contexte où la flexibilité du système productif devient une donnée, la formation professionnelle est conçue comme un élément à intégrer dans des parcours professionnels individuels exposés aux risques du chômage. Ces derniers ne se limitent plus aux jeunes : l'élaboration de procédures permettant de garantir l'égalité d'accès à la formation professionnelle « tout au long de la vie » devient un enjeu d'équité pour les pouvoirs publics qui enjoignent les partenaires sociaux de remédier aux dysfonctionnements du système de formation professionnelle continue, les catégories les plus fragiles n'y ayant pas ou peu accès. 
Les dispositifs élaborés au cours de cette période sont donc fortement marqués par un contexte où la responsabilisation des individus sur leurs parcours devient primordiale. La loi du 4 juillet 1990 définit un droit individuel à la qualification s'appuyant sur un crédit formation individualisé (CFI) délimitant un dispositif d'accès à une formation diplômante à partir d'un bilan de compétences que le salarié ou le jeune pourra effectuer auprès d'un organisme accrédité. Cet outil permet d'individualiser les parcours de formation alors que le dispositif de 1970-1971 était le plus souvent conçu suivant une logique de stage destiné globalement et uniformément à un ensemble de salariés. L'accord interprofessionnel de juillet 1991, repris dans la loi de décembre 1991, poursuit cette logique d'individualisation en introduisant le bilan de compétences et la possibilité d'un co-investissement entre l'entreprise et le salarié : une partie de la formation (25\%) peut être effectuée hors temps de travail, à condition que celle-ci soit une formation longue, qualifiante sinon diplômante. L'entreprise s'engage à examiner les possibilités d'une reconnaissance à l'issue de la formation. Du fait de cet encadrement, les organisations syndicales, à l'exception de la CGT, souscrivent à cette possibilité. Celle-ci sera peu utilisée. Mais son introduction dans le dispositif de 1991, conjointement à d'autres éléments comme le droit au bilan de compétences tous les cinq ans ou la référence à une première version de la validation des acquis, entérinent dans la négociation les premiers éléments d'une gestion individualisée des parcours professionnels.

La gestion individualisée est au cœur de l'accord de 2003 (Maggi-Germain, 2009). Son objectif est de "permettre à chaque salarié d'être acteur de son évolution professionnelle ${ }^{9}$ en s'appuyant sur des dispositifs qui conduisent, selon l'un des négociateurs patronaux, à asseoir une véritable " personnalisation » de la formation. Cette dernière est instrumentée par l'instauration d'entretiens professionnels tous les deux ans et la généralisation du recours au bilan de compétences. L'accord se place sous le signe de la co-responsabilité : l'élargissement des possibilités de recours aux formations hors temps de travail sous-tend une conception dans laquelle l'employeur paie les frais de formation, le salarié « paie » de son temps et/ou de son salaire lorsqu'il veut maintenir ou développer son employabilité ${ }^{10}$. On peut également penser que l'accent mis sur la reconnaissance dans l'emploi et les classifications des formations qualifiantes, en particulier lorsqu'elles auront été suivies en dehors du temps de travail, s'inscrit dans la recherche de compensations positives aux efforts consentis par les salariés. L'Etat est également partenaire de cette co-responsabilité grâce aux exonérations de charges sociales et à l'engagement pris d'élaborer des mesures réservées aux salariés non qualifiés désirant acquérir un diplôme. La co-responsa-

9. Extrait du préambule de l'accord.

10. Ce terme n'est pas mentionné dans l'accord mais il est sous-jacent dans deux des catégories de la typologie retenue des actions de formation incluses dans le plan de formation. 
bilité est assortie d'une co-initiative, en particulier dans l'instauration du droit individuel à la formation (DIF), dont le salarié pourra avoir l'initiative mais pour lequel il devra obtenir l'accord de l'employeur. Cette condition limitative, ainsi que les restrictions posées à sa transférabilité, en font un droit tout à la fois attaché à la personne et un élément du contrat de travail. Elles le font apparaitre comme une tentative de surmonter la coupure entre le plan de formation (initiative de l'employeur) et le congé individuel de formation (initiative du salarié) à travers l'instauration d'un espace de co-initiative encadré collectivement par l'obligation de consulter le comité d'entreprise sur la mise en œuvre du DIF introduite par la loi de 2004 (Merle, 2004).

Les dispositifs de 1991 et 2003-2004 s'inscrivent ainsi dans une certaine continuité, le premier introduisant une approche individualisée de la formation que le second est venu consolider plus d'une décennie plus tard. Ils se placent dans un contexte d'enracinement dans la crise et ses caractéristiques de flexibilité et de précarité et dans un environnement où l'action des acteurs de la négociation et des politiques publiques aux différents niveaux dessinent une voie - souvent dénommée «troisième voie » (Giddens, 1998) - dont l'objectif est d'articuler flexibilité des entreprises et sécurité des individus. Dans cette optique, la responsabilité de ces acteurs est de construire les garanties collectives propres à encadrer ces processus d'individualisation en donnant aux individus les moyens de se prémunir contre les risques de l'emploi et en les équipant afin qu'ils soient à même de construire leurs trajectoires professionnelles dans et hors de l'entreprise. La fabrication dans les instances européennes des «idées floues » de formation et éducation tout au long de la vie (FETLV) (Verdier, 2008) ou encore de flexisécurité (ou flexicurité) (Gaudu, 2008 ; Verd, Vero, 2011) ouvre la voie à leur appropriation sous des formes à la fois spécifiques et diversifiées par les acteurs de la configuration sociale française : «Même si elle n'impose pas ni même n'incite, la stratégie européenne contribue de plus en plus à structurer les débats nationaux et inspire plus ou moins directement la définition des problèmes dont s'empare telle ou telle réforme nationale » (Verdier, 2008:130). La « sécurité sociale professionnelle » prônée par la CGT, ou encore la "sécurisation des parcours professionnels » défendue par la CFDT, se placent dans les stratégies de négociation de consolidation des droits au cours des étapes de la vie professionnelle dans et hors de l'entreprise, afin d'aller vers un nouveau statut de travail salarié pour la première ou de négociation plus locale ou professionnelle de parcours pour la seconde. Elles s'inscrivent dans les traditions de ces organisations mais également dans des évolutions nées de leur participation aux débats européens. A bien des égards, l'accord de 2003 parait se placer dans le sillage du «cadre d'action pour le développement des compétences et 
des qualifications tout au long de la vie » adopté par les partenaires sociaux européens en février 2002, qui affirme ce principe de co-responsabilité : à l'entreprise de faire en sorte que le développement des compétences des salariés soit un enjeu de sa performance, au salarié que le développement des compétences soit un enjeu de la conduite de son parcours professionnel, à l'Etat et aux collectivités locales de favoriser les possibilités d'apprentissage pour plus de compétitivité et de cohésion sociale. La CGT, qui n'avait pas signé l'accord de 1991 car elle refusait le principe du co-investissement, appose sa signature sur celui de 2003. Dans l'intervalle, le contexte économique et social s'est profondément transformé ; cette organisation a adapté sa stratégie politique et revendicative à ces mutations. Mais elle a été aussi partie prenante, comme les autres organisations professionnelles et syndicales, de la construction des référentiels d'action publique, notamment à l'échelle européenne, dans les deux dernières décennies.

\section{I.2. Des dynamiques d'acteurs fortement ancrée dans la concertation}

Le domaine de la formation professionnelle continue (FPC) est analysé comme le champ privilégié du consensus et de la négociation sociale. Les travaux de Tanguy $(1999$; 2001) ont montré que ce domaine est construit à la Libération comme à la fois un lieu d'entente sociale, un instrument de changement et de modernisation sociale, et qu'il est fondé sur un principe d'équité ; les groupes porteurs sont précisément des « conciliateurs, des hommes de compromis ». En ce qui concerne la négociation, d'autres travaux ont approfondi l'analyse historique des moments fondateurs de ce qui deviendra un système de négociation collective (Nallet, 1991 ; Vincent, 1997) en insistant sur le rôle de mai 1968 dans cette dynamique. Le système d'acteurs porte en effet la marque de cette mobilisation : la négociation est actée dans le constat de Grenelle et l'actualité du mouvement social remet sur le devant de la scène la nécessité de modifier la logique de conflit alors prédominante. Selon l'initiateur de la loi de 1971, les caractéristiques de l'enjeu formation sont contradictoires : elles relèvent tout à la fois du « domaine de la division irréductible » en ce qu'il touche aux prérogatives de gestion des directions d'entreprises et de celui des « convergences possibles » en termes d'attentes des salariés et d'élévation possible des qualifications (Delors, 1976/1991). Elles autorisent la recherche du compromis et du bien commun et justifient que la négociation d'un accord interprofessionnel précède l'adoption d'une loi qui en reprend plus ou moins complètement les dispositions. Pour les organisations professionnelles et syndicales - désignées dans ce domaine plus qu'ailleurs « partenaires sociaux »-, cette place particulière de l'enjeu formation plaide pour la constitution de la formation en sphère de régulation paritaire essentiellement autonome. La célébration récente des 40 ans du paritarisme est venue rappeler leur 
attachement à ce système et à ses capacités d'adaptation. L'articulation de la négociation interprofessionnelle et de la loi qui marque ce domaine de sa fondation en 1970-1971 jusqu'à nos jours - à l'exception d'une courte période en 1993 au cours de laquelle le gouvernement, à travers la loi quinquennale sur l'emploi, fixe clairement l'agenda de la négociation - y était donc inscrite dès l'origine. Ces conditions d'émergence du système éclairent l'origine d'un certain nombre de représentations sur ce domaine de négociation. Ainsi la FPC a été considérée tour à tour comme le « jardin » des partenaires sociaux ${ }^{11}$, comme l'objet idéal-typique de la « loi négociée » (Verdier, Langlois, 1972) ou comme le lieu où s'est construit un véritable « régime paritaire » (CAS, 2007).

\section{Une sphère paritaire sous domination patronale jouissant d'une autonomie encadrée}

Un certain nombre de caractéristiques du système semblent accréditer son caractère paritaire. Des organismes de collecte dotés de conseils d'administration paritaire depuis 1994 gèrent des fonds importants; dans les périodes comprises entre les négociations interprofessionnelles, le système est piloté par le comité paritaire national pour la formation professionnelle (CPNFP) réunissant l'ensemble des organisations professionnelles et syndicales ${ }^{12}$. De l'avis de la plupart des négociateurs, cet organe a un rôle majeur dans la mesure où y sont élaborées des réflexions voire des décisions qui donneront lieu ensuite à des négociations. Le rôle d'orientation politique de cette instance est renforcé dans l'accord de 2009. Un des enjeux de la signature unanime de l'accord de 2003 était précisément l'intégration de la CGT dans cet organe de gouvernance, assurant ainsi sa présence dans le «club » des spécialistes en charge de garantir la pérennité du système paritaire de la formation professionnelle continue. Enfin, ce « régime paritaire » est assez puissant puisqu'il gère $40 \%$ des fonds de la formation professionnelle. Toutefois, l'hégémonie patronale ou encore le rôle de l'Etat dans la régulation du système et dans la co-construction des normes interrogent le paritarisme de cette sphère de régulation.

L'hégémonie patronale est assurée par le rôle prédominant que joue la principale organisation patronale dans ces organismes paritaires : elle en détient le secrétariat, qu'il s'agisse du CPNFP ou encore du Fonds unique de péréquation (FUP) ${ }^{13}$; elle a imposé le système de délégation de gestion à une association patronale dans la plupart des organismes paritaires collec-

11. Expression d'André Ramoff, délégué à la formation professionnelle dans les années 1980, cité par Mériaux (1999).

12. Cette instance a été créée par l'ensemble des organisations signataires de l'accord de 1991.

13. Le Fonds unique de péréquation (FUP) assurait la péréquation entre les fonds issus de la cotisation des entreprises relatives à la professionnalisation et ceux relatifs au congé individuel de formation, l'information sur l'utilisation des cotisations relatives à ces deux obligations ainsi que l'animation du réseau des collecteurs du congé individuel de formation. Il a été remplacé dans l'accord de 2009 par le FPSPP (Fonds paritaire de sécurisation des parcours professionnels) (infra). 
teurs agréés (OPCA) (Mériaux, 1997 ; Luttringer, 2008). Comme l'indique Freyssinet (2010:31) après une analyse d'un demi-siècle de négociations sur l'emploi, « le patronat privilégie l'exercice d'une capacité de régulation autonome des partenaires sociaux dès lors qu'il en a l'initiative et qu'il s'estime capable d'en garder le contrôle ». Ce contrôle est d'autant plus effectif que la négociation se fait à partir d'un projet patronal, élaboré sous la houlette du CNPF puis du MEDEF, qui fixe l'unité de la parole patronale. Celle-ci a longtemps été assurée par la domination des grandes fédérations de la métallurgie et du bâtiment dans les commissions d'élaboration des projets. Ces fédérations, adhérentes à la fois à la CGPME et au CNPF/ MEDEF, pouvaient en outre influer sur ces deux organisations. L'expertise en matière de formation était largement concentrée à l'UIMM : son délégué général adjoint, dont les compétences étaient largement reconnues, a imposé ses vues à l'ensemble du camp patronal - et l'intérêt de la métallurgie comme étant celui de l'ensemble du patronat - durant près de vingt ans (entre 1987 et 2005). Ainsi s'est forgée la place revendiquée de " pilote du paritarisme ${ }^{14}$ » tenue par le CNPF puis le MEDEF.

L'autonomie de cette sphère est également interrogée par le rôle direct ou indirect que tient la puissance publique dans le fonctionnement du système et dans l'élaboration des normes. A cet égard, les processus à l'œuvre dans la FPC ne sont pas différents de ceux existant dans d'autres champs $\mathrm{du}$ paritarisme : ils permettent de souligner l'autonomie, mais non l'indépendance, qu'ont les partenaires sociaux vis-à-vis des pouvoirs publics (apRoberts et al., 1997:21), ou de qualifier les systèmes paritaires de «fragments néo-corporatistes » (Duclos, Mériaux, 1998). Ces dernières analyses cernent également les processus d'échange politique qui se déploient dans le domaine de la FPC, dans lesquels la reconnaissance institutionnelle des acteurs - et les ressources qui lui sont adjointes - s'échangent contre la contribution du système à la réussite des objectifs de la politique publique (Mériaux, 1999).

L'analyse comparée du contenu des accords et des lois ou encore des conditions de négociation (Tallard, 2004) confirme l'encadrement de cette autonomie revendiquée par les partenaires sociaux. La comparaison des stipulations de l'accord de 1970 et des dispositions de la loi de 1971 (Vincent, 1997) permet de montrer, par exemple, qu'alors même que la négociation sur le mode de gestion des associations de formation avait échoué du fait de la division syndicale et du refus patronal de tout ce qui pouvait se rapprocher de la co-gestion, la loi ouvre la possibilité d'une gestion paritaire des fonds dans une optique d'assurance contre les risques d'inadaptation professionnelle. C'est donc bien une régulation partagée qui se met en

14. Cette expression est revenue souvent dans nos entretiens, que ce soit avec les représentants du MEDEF national ou avec ceux des principales fédérations. 
place dès l'instauration de l'obligation de financement. De même, quelques années plus tard, après la négociation de 1983, la loi de 1984 ne reprend pas un certain nombre de dispositions élargissant le rôle des commissions paritaires nationales de l'emploi (CPNE) en matière d'élaboration des certifications ${ }^{15}$ et elle introduit une obligation de négocier sur les objectifs et les moyens de la formation. Enfin, avant la plupart des négociations, que l'Etat incite le plus souvent à engager, sont réunis à l'initiative des pouvoirs publics des commissions tripartites donnant lieu à l'élaboration de rapports, versés plus ou moins directement à la négociation ${ }^{16}$. Se forge ainsi un langage commun qui sera ensuite décliné avec une certaine autonomie dans les négociations. Il constitue le lexique des négociations, qui peuvent être âpres comme le montre la difficulté d'aboutir à des accords unanimes ou encore la rupture des négociations en 2001.

\section{La consolidation du rôle des branches professionnelles}

Malgré la création des CPNE par l'accord sur la sécurité de l'emploi de 1969, qui témoigne de la volonté de mettre en place au niveau des branches professionnelles une instance de suivi des évolutions de l'emploi et de définition des actions de formation susceptibles d'accompagner les transformations techniques et organisationnelles, ces commissions seront en sommeil jusqu’au début des années 1980. Dans les premières années de fondation du système, les quelques organismes collecteurs professionnels avaient essentiellement une fonction technique de gestion, de conseil dans l'élaboration des demandes ou encore de réponse ponctuelle. Hormis dans quelques activités impliquées de longue date dans l'apprentissage (BTP, services de l'automobile), les négociations de branches ne traitaient de la formation qu'en ce qui concerne les modalités de reconnaissance des diplômes dans les grilles de classification. L'accord de 1983 sur l'alternance introduit une rupture en impliquant les CPNE dans la définition des priorités dans le cadre des contrats de qualification et en leur donnant la possibilité de mettre en place, dans ce but, des certifications spécifiques. Au fil des dispositifs, c'est la possibilité de définir de véritables certifications professionnelles qui leur incombe. Le rôle des branches professionnelles est également consolidé par la possibilité de mettre en place des collecteurs de la contribution sur l'alternance et l'introduction de la négociation obligatoire sur les objectifs et les moyens de la formation dans la loi de 1984.

15. L'enjeu était alors de maintenir le monopole de l'Education nationale sur la construction des qualifications et ce ministère a réussi, un temps, à imposer son point de vue en s'appuyant sur les tensions au sein même du ministère du Travail entre la Direction des relations du travail (DRT) attachée à la préservation des garanties des salariés (et par là aux formations qualifiantes) et la Délégation à la formation professionnelle (DFP), plus à l'écoute des demandes des entreprises.

16. Guilloux (1996) qualifie d'" accord pré-négocié " l'accord interprofessionnel du 21 septembre 1982 sur le congé individuel de formation dont la négociation a été précédée de plusieurs groupes de travail quadripartites (Etat, organisations d'employeurs, organisations syndicales, experts) et qui s'ouvre sur la base de l'hypothèse du rapport de synthèse d'un groupe qui avait la préférence du ministre. 
Le dispositif de 1991 élargit encore les prérogatives des CPNE et renforce l'obligation de négocier dans la branche. La réforme des organismes de collecte, imposée par les pouvoirs publics en 1994, a permis d'accroître la capacité d'action de ces organismes et les moyens dont disposaient les branches professionnelles pour élaborer et mettre en œuvre leurs politiques de formation. Une décennie plus tard, le dispositif de 2003-2004 renforce le rôle de la négociation de branche en réduisant de cinq à trois ans sa périodicité et en élargissant les thèmes à traiter à la mise en œuvre des dispositions de l'accord et notamment le droit individuel à la formation (DIF). Il accroît également le poids institutionnel des branches en élargissant les prérogatives des CPNE et en les dotant de capacités d'expertise à travers l'obligation de créer des observatoires prospectifs des métiers et des qualifications, amplifiant ainsi la tendance des branches professionnelles à devenir des centres de ressources pour les entreprises (Morin, 1996).

\section{La montée en puissance des régions à l'initiative des pouvoirs publics}

La négociation collective interprofessionnelle se place traditionnellement dans une logique professionnelle et prend peu en compte la logique territoriale. De plus, dans la mesure où les normes conventionnelles sont rarement définies à ce niveau ${ }^{17}$, les acteurs parties prenantes de la négociation, qu'ils soient patronaux ou syndicaux, ont longtemps eu des difficultés à s'y structurer (Vincent, 2002). Tout en proclamant une volonté d'articuler logique professionnelle et logique territoriale, les accords interprofessionnels ont toujours échoué à atteindre cet objectif et cet échec a généralement été considéré comme la principale faiblesse de l'accord de 2003 (Merle, 2004). Le rôle croissant des régions en matière de formation - qui conduit certains auteurs à s'interroger sur une inflexion du modèle français de formation professionnelle d'une logique professionnelle vers une logique territoriale (Géhin, 2002) - est donc le résultat des initiatives des gouvernements successifs : en témoignent les lois de décentralisation de 1983 et 1985, la loi quinquennale sur l'emploi de 1993 et les dispositifs législatifs de la décennie 2000 qui organisent de nouvelles dévolutions aux régions en matière de formation professionnelle. Toutefois, l'intervention des régions, si elle représente près de $40 \%$ des dépenses de formation, est essentiellement consacrée à l'apprentissage et à l'action vers les demandeurs d'emploi ; celle en direction des salariés est minoritaire et prend essentiellement la forme des contrats d'objectifs territoriaux dans lesquels la région abonde les financements des OPCA de branche sur des objectifs prioritaires définis en commun.

17. Un mouvement de déclin des conventions collectives départementales s'est amorcé dans les années 1970 ; à quelques exceptions près, elles ne sont plus actives que dans la Métallurgie ou le BTP. 
Cette analyse à grands traits de la négociation collective sur la formation professionnelle continue entre 1970 et 2003 permet de saisir, à l'aube des années 1990, la transformation des enjeux d'une vision collective d'adaptation aux transformations des emplois prédominante depuis la fin des années 1960 à une vision plus individualisée de sécurisation des parcours professionnels. Elle permet également de cerner les principales caractéristiques de la «scène » de la négociation au milieu des années 2000 : c'est un système paritaire par les institutions qu'il gère, et dont le fonctionnement est dominé par l'acteur patronal ; en outre, ce système est régi par des règles issues de la négociation collective, qui sont insérées dans des logiques d'action publique à différentes échelles qui influencent la formulation des enjeux et les contenus des dispositifs négociés. Peut-on analyser l'accord de 2009 en s'appuyant sur ces caractéristiques?

\section{L'accord de janvier 2009, une négociation contrainte, un contenu limité ?}

Alors que l'accord interprofessionnel sur la formation professionnelle tout au long de la vie de 2003 a souvent été désigné comme " historique » et de nature à modifier les nombreux dysfonctionnements du système de formation professionnelle continue, le nouveau président de la République qualifie en juillet 2007 le système de formation professionnelle de « système à bout de souffle » qu'il y aurait lieu de profondément réformer. Pour autant, les multiples acteurs mobilisés au cours du processus de négociation s'accordent pour estimer que l'accord de janvier 2009 n'est qu'un accord de gouvernance peu novateur quant à son contenu et que le " grand soir » de la formation professionnelle n'a pas eu lieu. Ce résultat décevant serait à mettre au crédit d'un trop grand interventionnisme des pouvoirs publics, qui aurait conduit les acteurs du système paritaire à se coaliser pour le maintenir face aux attaques dont il était l'objet. Au-delà des polémiques, on s'interrogera dans cette partie sur la continuité ou la rupture des dispositifs négociés en analysant les dynamiques de négociation et le contenu de l'accord de 2009.

\section{II.1. Un interventionnisme étatique hors du commun ?}

L'analyse de la période précédente a mis en évidence une co-construction des règles du système entre l'acteur public et les partenaires sociaux. C'est donc à cette aune qu'il faut examiner le rôle de chacun des acteurs dans la négociation de l'accord de 2009.

L'intervention publique se place dans un double contexte. La loi du 31 janvier 2007 sur la modernisation du dialogue social modifie le paysage de la négociation collective en instituant des procédures visant à une 
programmation annuelle des réformes envisagées dans le domaine social et à l'ouverture d'une négociation avant toute intervention législative dans ce champ. A cet effet est prévue une période de concertation et la transmission par le gouvernement d'un document d'orientation " présentant des éléments de diagnostic, les objectifs poursuivis et les principales options ${ }^{18}$. Les partenaires sociaux font alors connaitre leur volonté de négocier et le délai qui leur paraît nécessaire. Dans le cas des négociations de 2009, cette intervention des pouvoirs publics est précédée d'un rapport d'information sénatorial publié début juillet 2007. Il stigmatise l'enlisement des priorités du système de formation professionnelle et préconise des réformes radicales telles que la reconfiguration des OPCA, la suppression de l'obligation légale concernant le plan de formation ou encore un pilotage territorial des politiques de formation professionnelle confiée aux régions ${ }^{19}$. C'est largement sur ce diagnostic que s'appuie l'interpellation du président de la République en juillet 2007. Cependant, ce scénario n'est pas très différent de ceux qui ont précédé d'autres négociations : rapports d'experts ou d'organes d'inspection, commandés par les pouvoirs publics, fort critiques quant à son fonctionnement, au manque de transparence de ses circuits de financement ou encore son incapacité à faire face aux inégalités d'accès à la formation, qui se sont souvent concrétisées dans des injonctions à négocier des réformes du système. Le volontarisme est donc certes marqué dans la forme. Mais l'enchaînement des évènements n'est pas entièrement nouveau compte tenu des nouvelles contraintes imposées par la loi de modernisation sociale.

L'intervention des partenaires sociaux s'insère dans un calendrier programmé dans d'autres négociations. La négociation de l'accord de 2009 prend place quelques mois après la conclusion de l'accord sur la modernisation du marché du travail. Ce dernier, considéré par Freyssinet (2010:230) comme exemplaire d'« un accord multidimensionnel sur l'emploi », repose fortement sur l'idée de construction de parcours professionnels qu'il convient d'accompagner quel que soit le statut indemnitaire du salarié (salarié ou demandeur d'emploi) pour assurer au mieux les phases de transition sur le marché du travail. Cet accord élargit les conditions de portabilité du DIF et fait référence, dans son article 15, à une négociation à venir sur la formation au cours de laquelle serait posée la question de l'ouverture éventuelle des fonds de la formation professionnelle des salariés aux demandeurs d'emploi pour assurer le financement de la qualification ou la requalification des demandeurs d'emploi les plus éloignés de l'emploi. Sont ainsi posés les jalons d'une prise de distance avec la séparation étanche entre le système destiné aux salariés et celui en direction des demandeurs

18. Extrait de l'article L. 101-1 modifié du code du travail.

19. Jean-Claude Carle, Rapport d'information du Sénat sur le fonctionnement des dispositifs de formation professionnelle, juillet 2007. 
d'emploi ${ }^{20}$. Par ailleurs, les négociateurs de l'accord national interprofessionnel (ANI) de 2003 avaient convenu dans l'article 28 de cet accord de tirer un bilan de sa mise en œuvre après cinq ans afin d'examiner les éventuelles évolutions nécessaires.

Les négociations qui s'ouvrent en septembre 2008 se placent donc, dans une certaine mesure, dans une double dynamique initiée par les partenaires sociaux et dans le nouveau décor dressé par la loi de 2007. Toutefois, les pouvoirs publics feront une interprétation extensive du processus de concertation sociale prévu par la loi de 2007 en instituant deux groupes de travail, l'un tripartite - au sein du Conseil d'orientation pour l'emploi (COE) ${ }^{21}$ pour établir un diagnostic - puis le second quadripartite - incluant les régions - et destinés à préparer le document d'orientation. Ce groupe multipartite est donc chargé d'un travail politique de clarification des priorités et des moyens de la réforme tant dans ses modalités de financement que dans la gouvernance du système. Du côté des partenaires sociaux, les participants à ce groupe sont des responsables susceptibles d'engager leurs organisations, certains d'entre eux ayant été des négociateurs de l'accord de 2003. Les pouvoirs publics, représentés tant par des directions techniques que par des cabinets ministériels n'ont, selon la plupart des protagonistes des groupes de travail, pas de stratégie politique affirmée en dehors de l'objectif principal de faire contribuer les fonds de la formation professionnelle à la politique d'emploi par la création d'un fonds tourné vers les demandeurs d'emploi. La Direction générale de l'emploi et de la formation professionnelle (DGEFP) a une analyse fondée sur une évaluation des insuffisances de l'accord de 2003 et des propositions précises, concernant notamment la mise en place de fonds régionaux de sécurisation des parcours professionnels. Cette proposition apparait toutefois comme ayant un statut ambigu car elle émane de l'administration, tout en n'étant pas explicitement soutenue par le cabinet du ministre. Enoncée dès le début des travaux du COE, puis reprise lors de ceux du groupe multipartite, cette proposition est perçue de fait comme celle des pouvoirs publics. Les régions s'y opposent : elles y voient une tentative de l'Etat de les déposséder de leurs prérogatives en matière de formation. Le document final propose trois grandes orientations stratégiques partagées - améliorer l'efficacité du système, le rendre plus équitable, le simplifier - déclinées en huit axes principaux de réforme pour chacun desquels sont listés les termes du débat, les consensus, les alternatives et thèses en présence,

20. Pour les négociateurs, il s'agit d'une réforme fondamentale dans la mesure où elle modifie l'approche uniquement statutaire (salarié/demandeurs d'emploi) qui prévalait à l'origine en matière de formation professionnelle.

21. Le Conseil d'orientation pour l'emploi (COE) est un organisme créé par décret en avril 2005. II est constitué de représentants des partenaires sociaux, des parlementaires, du Conseil économique et social, des collectivités territoriales (Association des maires de France, Association des régions de France, Association des départements de France) ainsi que de personnalités qualifiées. 
les scénarios d'évolution ou de réforme et éventuellement les questions supplémentaires méritant un examen approfondi. Une telle présentation constitue une demande forte des organisations patronales, soucieuses de faire montre de leur autonomie en laissant le plus ouvert possible le champ de la négociation.

S'appuyant sur les travaux du groupe multipartite, le gouvernement transmet fin juillet aux partenaires sociaux un document d'orientation fixant quatre objectifs prioritaires et détaillant les thèmes et la liste des points à aborder dans la négociation interprofessionnelle. Comme le fait remarquer Freyssinet (2010), ce document est étonnamment précis et directif par rapport à ceux diffusés préalablement à d'autres négociations qui se déroulent au même moment. De plus, après l'ouverture des négociations, l'intervention des pouvoirs publics ne se cantonne pas à une pression sur le calendrier mais les ministres interviennent dans la presse pour influencer le cours même des négociations, suscitant l'irritation des négociateurs ${ }^{22}$.

L'interventionnisme des pouvoirs publics n'est pas négligeable. Toutefois, au regard de leur faible capacité de proposition dans le déroulement des groupes de travail et de l'insertion de l'accord dans des dynamiques déjà enclenchées par l'accord de janvier 2008 ou celui de 2003, on peut se demander s'il ne s'agit pas surtout de mettre en scène le rôle dominant et réformateur de la puissance publique par rapport à celui de partenaires sociaux attachés à la conservation de leurs privilèges au sein du système paritaire.

\section{II.2. La difficulté à construire une parole patronale}

Le processus de préparation de la négociation se déroule quelques semaines après la mise à jour du « scandale des fonds secrets de l'UIMM » en octobre 2007, qui vient particulièrement déstabiliser le domaine de la formation. La figure traditionnelle d'une parole patronale unifiée sous l'égide de l'UIMM a commencé à se lézarder dès 2005. En effet, l'arrivée à la tête du MEDEF de Laurence Parisot, portée par les territoires et les fédérations des services, a entraîné la nomination d'un représentant de ces mêmes territoires à la tête de la commission formation. Celui-ci a rapidement pour objectif de reconquérir, non sans difficultés, une certaine autonomie de décision face aux représentants de l'UIMM qui détenaient les postes clés et étaient porteurs d'un fort niveau d'expertise et d'influence tant en interne qu'auprès des pouvoirs publics. Cet affaiblissement du poids de l'UIMM au sein de la commission formation du MEDEF - et donc de sa part dans l'élaboration de la stratégie du MEDEF - ainsi que le repli amorcé de cette

22. Dans une déclaration commune adressée au gouvernement dans les premières semaines de la négociation, l'ensemble des partenaires sociaux réaffirment leur attachement à l'autonomie de la négociation collective et leur souhait d'inscrire cette négociation dans la logique de celles déjà engagées suite à l'accord de janvier 2008. 
fédération sur des négociations propres à la métallurgie ne peuvent manquer de libérer des paroles qui jusque-là n'avaient pas trouvé la possibilité de s'exprimer tant au sein du MEDEF que dans les autres organisations patronales. Ainsi, l'Union des industries textiles, qui représente des PME évoluant sur un marché ouvert très concurrentiel, affirme dès septembre 2007 qu'il faut réformer le système pour recentrer les dispositifs de formation sur les salariés peu qualifiés; de con côté, la Fédération de la formation professionnelle (FFP), pour laquelle l'existence des OPCA constitue une concurrence déloyale, demande plus de transparence et de lisibilité dans les mécanismes de financement de la formation professionnelle et une amélioration du fonctionnement des OPCA. A la CGPME, où l'UIMM est aussi un acteur majeur, une volonté d'autonomie se concrétise dès la fin de l'année 2006 avec la nomination d'un nouveau président chargé de conduire les travaux d'une commission formation-éducation plus restreinte dans laquelle branches et régions sont représentées à parts égales ${ }^{23}$.

A l'automne 2007, la crise de l'UIMM se traduit par le retrait des mandats MEDEF de ses responsables et la mise à l'écart définitive des animateurs historiques du domaine de la formation professionnelle. La commission formation se trouve donc en partie privée de ses personnalités dominantes et des capacités d'expertise qu'elles représentaient. Parallèlement, la direction de la délégation patronale à la négociation sur la modernisation du marché du travail est retirée à l'UIMM pour être confiée à une personnalité issue des services. Dans un tel contexte, tout au long du processus de préparation de la négociation au sein des groupes de travail puis au cours de la négociation, le MEDEF éprouve des difficultés à construire une ligne politique unifiée face à la difficile articulation entre logique de branche et logique territoriale et aux intérêts divergents de ses fédérations. Ceux-ci reflètent souvent le clivage entre PME et grandes entreprises ou encore les intérêts particuliers de certaines activités pour lesquelles le système de collecte paritaire constitue un obstacle à leur développement.

La crise de l'UIMM s'est aussi traduite par des changements profonds dans l'animation par le MEDEF de la délégation patronale à la négociation sur la formation professionnelle. Le nouveau délégué général de cette organisation a conduit la délégation patronale. Cependant, de l'avis de la plupart des négociateurs, soucieux de se forger une nouvelle légitimité, il a fait montre d'une attitude particulièrement consensuelle, assumant davantage un rôle de chef d'orchestre que celui de seul représentant des intérêts de la métallurgie ${ }^{24}$.

23. Bien que conviée, I'UIMM ne siège pas dans cette instance. Toutefois, contributeur important au sein de la CGPME, cette structure est régulièrement informée et consultée sur la teneur des propositions faites.

24. La gouvernance de I'UIMM, notamment en ce qui concerne le domaine de la formation professionnelle, a été profondément transformée pour laisser une place plus importante à la représentation des territoires par rapport à celle des grandes entreprises auparavant prédominante. 
Toutefois, compte tenu de la fragilité du MEDEF et de la volonté d'affirmation de la CGPME dans ce nouveau contexte, les relations entre la CGPME et le MEDEF sont difficiles tout au long de la négociation. Audelà des stratégies de positionnement de chacun au moment où la question de la représentativité des organisations professionnelles et syndicales est en débat, il y a de vrais sujets de divergence : le lien à maintenir entre individualisation des droits et leur encadrement collectif, ou la suppression de l'obligation légale pour lui substituer des obligations de nature conventionnelle en font partie. Sur le premier sujet, la CGPME, dans le but de séparer plus clairement les formations relevant selon elle de l'initiative individuelle et celle du domaine du plan de formation, propose la fusion entre le DIF et le CIF. Pour le MEDEF, il est nécessaire de maintenir le lien à l'emploi contenu dans le CIF et celui à l'entreprise formalisé dans le DIF. D'autant que ce dernier est déjà fortement intégré dans les pratiques de gestion des ressources humaines des grandes entreprises. Le MEDEF réussit à écarter cette proposition en s'appuyant sur l'opposition résolue des organisations syndicales à cette fongibilité qui, pour elles, dénature le CIF et s'écarte de la conception du salarié acteur de son projet inscrite dans l'accord de 2003. Quant à la remise en question de l'obligation légale, si elle est poussée par les travaux du groupe multipartite, la CGPME, pour laquelle la préservation de l'existence de l'Association de gestion des fonds salarié des petites et moyennes entreprises (AGEFOS-PME) est indispensable à la sienne propre, y est farouchement hostile et elle ne fait pas consensus au sein des différentes fédérations patronales du MEDEF. L'UIMM, voulant maintenir et développer son appareil de formation et son OPCA, y est totalement opposé alors que la FFP, dont l'objectif est la disparition des OPCA, souhaite sa suppression. En ce qui concerne les organisations syndicales, si la CFDT, attachée à la primauté du contrat sur la loi, y serait favorable sous certaines conditions, ce point fait l'objet d'un refus catégorique des autres organisations syndicales qui y voient un risque d'éclatement des droits. La priorité au maintien de l'unité du monde patronal et l'objectif d'aboutir à une signature unanime de l'accord conduiront à écarter cette proposition.

Le déroulement de la négociation de 2009 est donc marqué par une certaine fragilisation de la domination traditionnelle du MEDEF sur l'acteur patronal dans une situation où les organisations syndicales attendent des propositions unifiées. Elle s'inscrit également dans une mise en cause publique du système paritaire à travers les tentatives des pouvoirs publics d'intervenir dans les négociations. La conjonction de ces deux facteurs a conduit les négociateurs à écarter les réformes radicales qui risquaient d'accroître la déstabilisation du système et à inscrire les dispositifs négociés dans la continuité de ceux initiés dans l'accord de janvier 2008. 


\section{II.3. Un accord qui marque l'aboutissement de la connexion entre le champ de l'emploi et celui de la formation professionnelle}

A bien des égards, l'accord de 2009 constitue l'aboutissement des tendances engagées depuis 1991. La conception de l'individualisation de la formation, qui s'accentue dans les dispositifs successifs depuis 1991, sous-tend en effet une logique d'instrumentation de la formation dans la construction de parcours professionnels dans lesquels se succèdent des périodes d'activité et de transition entre deux emplois qui peuvent être marquées par le chômage. Dès lors, la pérennité des droits individuels attachés à l'emploi et les frontières entre les circuits de financements de la formation relevant respectivement des statuts de salarié et de celui de demandeur d'emploi ainsi que l'efficacité des organisations en charge de la gestion de ces financements sont réinterrogés. Le système d'acteurs est également questionné par cette nouvelle optique s'inscrivant dans une vision de sécurisation des trajectoires professionnelles, qu'il s'agisse de l'action des organismes collecteurs ou de la place des régions. Le poids croissant des régions en matière de formation professionnelle, en particulier en ce qui concerne les demandeurs d'emploi ou les salariés en activité menacés dans leur emploi, en font en effet des acteurs incontournables que les négociateurs ne peuvent plus ignorer. Enfin, la logique d'individualisation implique aussi de mettre en place des outils susceptibles de faciliter les transitions et d'équiper l'individu afin qu'il puisse évaluer et faire valoir l'intégralité de ses capacités et se repérer dans la qualité de l'offre de formation et de certification.

Poursuivant dans cette voie d'une plus grande connexion entre le champ de l'emploi et celui de la formation professionnelle ouverte par l'accord de janvier 2008, les dispositifs négociés dans l'accord de 2009 tentent de répondre à ces multiples objectifs (encadré 2). La plupart des dispositions s'inscrivent dans cette logique d'organisation des transitions professionnelles, qu'il s'agisse de la simplification des catégories d'action entrant dans le cadre du plan de formation, de l'organisation du financement de la portabilité du DIF, de la création du FPSPP, de l'instauration de la "préparation opérationnelle à l'emploi » (POE). Ces dispositions, reprises et amplifiées dans la loi, pourraient conduire à « une annexion progressive du champ de la formation à celui de l'emploi ${ }^{25}$ » dans la mesure où les logiques et les règles de gouvernance dans ces deux champs tendent à se rapprocher.

Ces transformations sont accompagnées par une réforme de la gouvernance du système. Celle-ci clarifie les attributions des instances de branche et interprofessionnelles (Commissions paritaires nationales pour l'emploi (CPNE), Commissions paritaires interprofessionnelles régionales pour l'emploi (COPIRE)) et leur rôle de coordination avec les instances

25. Intervention de J.-L. Dayan à la table ronde de clôture du colloque « Emploi, compétences et relations professionnelles » (Paris, Université Paris-Dauphine, 24, 25 janvier 2011). 


\section{Présentation synthétique de l'Accord national interprofessionnel du 7 janvier 2009}

Plan de formation (art. 1 à 6). Deux catégories d'actions de formation sont distinguées: les actions d'adaptation au poste de travail et celles liées à l'évolution ou au maintien dans l'emploi dans l'entreprise, qui seront réalisées pendant le temps de travail ; les actions liées au développement des compétences, qui pourront être réalisées hors temps de travail.

Professionnalisation (art. 7 et 8). Le contrat de professionnalisation peut prévoir un tutorat externe pour les personnes de faible niveau de qualification ou les plus éloignées de l'emploi.

Portabilité du DIF (art. 11 à 16). En cas de rupture de contrat de travail, le salarié peut mobiliser le solde de ses droits à DIF pour contribuer au financement de formations réalisées pendant la période de prise en charge par l'assurance chômage ou dans les deux ans suivant son embauche par un nouvel employeur.

Préparation opérationnelle à l'emploi (POE) (art. 20 à 28). La POE permet de faire bénéficier un demandeur d'emploi, susceptible d'être recruté par une entreprise sur un emploi correspondant à une offre déposée à Pôle emploi, de la formation nécessaire.

Fonds paritaire de sécurisation des parcours professionnels (FPSPP). Le fonds unique de péréquation devient le Fonds paritaire de sécurisation des parcours professionnels. II a vocation notamment à abonder le financement d'actions de qualification et requalification au profit de salariés en déficit de formation et de demandeurs d'emploi.

Source : Liaisons sociales.

régionales ainsi que leur mode de fonctionnement et donne un poids plus important au CPNFP qui devient l'organe politique chargé d'élaborer les priorités d'action du FPSPP, lesquelles devront promouvoir des partenariats impliquant l'Etat, Pôle emploi et les instances régionales.

Dans un contexte où le système paritaire de la FPC était attaqué notamment par les pouvoirs publics et où le principal acteur patronal était fortement déstabilisé, l'ensemble des partenaires sociaux, forts de l'impact qu'avait eu la signature unanime de l'accord de 2003 et jugeant qu'il serait politiquement difficile pour le gouvernement de défaire significativement un tel accord lors de l'étape législative, ont choisi de faire bloc pour préserver le système et garder la maîtrise de ses évolutions. Les difficultés dans la négociation de cet accord sont plus venues des dissensions au sein de la délégation patronale qu'entre organisations patronales et syndicales. En ce qui concerne la création du Fonds de sécurisation des parcours professionnels, il se profilait déjà dans l'accord sur la modernisation du marché 
du travail de janvier 2008 et sa création est apparue au MEDEF comme inéluctable étant donné la pression gouvernementale. Dès lors, l'enjeu, que cette organisation a fait valider par l'ensemble des partenaires sociaux, était de garder le contrôle sur la gestion des fonds, c'est-à-dire de préserver son caractère paritaire ${ }^{26}$. En ce sens, le MEDEF est resté, tout au long de la négociation, le pilote du paritarisme.

L'élaboration de la loi - qui s'étalera sur près de dix mois - donne lieu à de nombreuses passes d'armes entre les parlementaires et le gouvernement, d'une part, et les partenaires sociaux, d'autre part. Une fois de plus, la légitimité des partenaires sociaux à créer de la norme et la place respective de la loi et du contrat dans cette construction sont réinterrogées ${ }^{27}$. De fait, si la loi reprend l'essentiel des dispositions de l'accord, elle y ajoute un contrôle de l'Etat sur les actions engagées à travers des conventions-cadre ou des conventions d'objectifs et de moyens passés tant avec le FPSPP qu'avec les OPCA. C'est ce même esprit de contrôle qui a présidé à l'écriture, non sans tensions, des décrets d'application. Pour Mériaux (1996), l'incapacité des acteurs à réformer les organismes de collecte pour les rendre plus efficaces en 1991 avait conduit l'Etat à reprendre la main par la loi quinquennale de 1993 et la réforme de la collecte qu'elle programmait (Mériaux, 1996). De même, on peut faire l'hypothèse que les effets limités des dispositions de l'accord de 2003 et la nécessité de faire montre d'une stratégie résolue face à l'acuité de la situation de l'emploi ont motivé, en 2008-2009, une volonté de prise de contrôle des pouvoirs publics sur la négociation et les mécanismes de la collecte à travers l'encadrement de la négociation et le conventionnement généralisé.

\section{Conclusion}

Ainsi, au regard des négociations menées depuis 1990, le dispositif de 2009 présente des caractéristiques contrastées. Il se place dans la continuité des premières par les enjeux d'individualisation qui y sont toujours dominants et dans le système d'acteurs marqué par l'hégémonie patronale et le poids de l'Etat. Mais les dispositions adoptées pourraient ouvrir de nouvelles voies au fonctionnement du système, en ce qui concerne tant la plus grande perméabilité entre les circuits de financements de la formation des demandeurs d'emploi et des salariés exposés aux risques du chômage

26. De même, face à une probable restructuration des OPCA sur une logique de surface financière, l'accord préconise des critères de regroupement s'inscrivant dans des logiques de proximité d'activité et tente de reprendre la main en proposant la création d'un groupe de travail chargé d'émettre des préconisations concernant ces regroupements.

27. Au lendemain de la signature de l'accord, le sénateur Carle, auteur du rapport de la mission d'information de juillet 2007, déclare que cet accord « manque de souffle et d'ambition » et demande au gouvernement et au législateur de " s'appuyer sur l'accord, l'accompagner et l'amplifier » (Inffo-flash, $\mathrm{n}^{\circ} 738,16-31$ janvier 2009, p. 12). 
que l'association de fait des régions et des acteurs qui s'y déploient, au travers des nombreux mécanismes de partenariat.

Plus généralement, l'examen sur longue période de la négociation collective en matière de formation professionnelle continue conduit à s'interroger sur la pérennité du système de négociation collective et de gestion paritaire institué en 1970-1971 et qui s'est développé au fil des décennies suivantes. Le passage à la formation professionnelle tout au long de la vie et la logique de construction des parcours professionnels qu'il sous-tend remettent en question la logique statutaire sur laquelle s'était construite le système de FPC, ainsi que la distinction traditionnelle entre le CIF relevant du projet du salarié et les dispositifs relevant de l'initiative de l'entreprise. Or cette coupure était à la base du système fondé en 1970-1971, lequel se trouve ainsi fragilisé (Join-Lambert, 2010). De plus, nous avons montré que les dispositions de l'accord de janvier 2009 viennent s'encastrer dans celles de l'accord de janvier 2008 sur la modernisation du marché du travail. Enfin, à des niveaux de branche ou d'entreprise, lors des négociations de GPEC, la formation professionnelle et ses dispositifs tendent à apparaittre comme de simples chapitres d'une vaste négociation sur l'emploi qui devient dominante dans la plupart des espaces de régulation (Didry, Jobert, 2010) : c'est donc bien la spécificité de cette négociation qui est désormais en cause, même si le système paritaire a pu s'assurer une certaine pérennité en acceptant de s'ouvrir à de nouveaux publics. 


\section{Références bibliographiques}

apRoberts L., Daniel C., Rehfeldt U., Reynaud E., Vincent C. (1997), « Formes et dynamiques de la régulation paritaire ", La Revue de l'IRES, $\mathrm{n}^{\circ} 24$, numéro spécial, Le paritarisme, institutions et acteurs, p. 19-42.

CAS (2007), "A quoi sert la formation professionnelle continue ? ", La Note de veille, $\mathrm{n}^{\circ} 62,11$ juin, p. 1-4.

Cannac Y. (1985), La bataille de la compétence, Paris, Editions Hommes et Techniques.

Commaille J. (2001), « La production des lois sur la formation permanente comme exemple de construction de la représentation du politique », Education permanente, $\mathrm{n}^{\circ} 149$, La formation permanente entre travail et citoyenneté, p. 29-40.

Commissariat général du Plan (2003), Gestion de l'emploi, gestion des compétences et formation professionnelle, Rapport du groupe de travail présidé par M.-L. Morin, mars.

David P.A. (1985), « Clio and the Economics of QWERTY », The American Economic Review, vol. 75, $\mathrm{n}^{\circ}$ 2, Papers and Proceedings of the Ninety-Seventh Meeting of the American Economic Association, p. 332-337.

Delors J. (1976/1991), « Genèse d'une loi et stratégie de changement », Connexions, $\mathrm{n}^{\circ} 17$, p. 39-50, repris dans Formation Emploi, $\mathrm{n}^{\circ}$ 34, p. 31-38.

Didry C., Jobert A. (dir.) (2010), L'entreprise en restructuration, dynamiques institutionnelles et mobilisations collectives, Rennes, PUR.

Dubar C. (1999), « De la "deuxième chance" au co-investissement : brève histoire de la promotion sociale (1959-1993) », in Dubar C., Gadéa C. (dir.), La promotion sociale en France, Villeneuve-d'Ascq, Presses universitaires du Septentrion, p. 31-49.

Dubar C. (2004), La formation professionnelle continue, $5^{\mathrm{e}}$ éd., Paris, La Découverte.

Duclos L., Mériaux O. (1998), « Le paritarisme, un fragment néo-corporatiste », in Auvergnon P., Martin P., Rozenblatt P., Tallard M., L'Etat à l'épreuve du social, Paris, Editions Syllepse, p. 219-229.

Dunlop J. (1958), Industrial Relations System, Southern Illinois University Press.

Freyssinet J. (2010), Négocier l'emploi. 50 ans de négociations interprofessionnelles sur l'emploi et la formation, Paris, Editions Liaisons.

Gaudu F. (2008), « De la flexicurité à la sécurité sociale professionnelle. L'emploi entre mobilité et stabilité », Formation Emploi, n 101, p. 71-88.

Géhin J.-P. (2003), « Les politiques régionales de formation : renforcement ou inflexion du modèle français de formation professionnelle ? ", in Bernier C., Jobert A., Rainbird H., Saglio J. (dir.), Formation, relations professionnelles et syndicalisme à l'heure de la société-monde, Paris, Québec, L'Harmattan, Les Presses de l'Université Laval.

Giddens A. (1998), The Third Way: The Renewal of Social Democracy, Londres, Polity Press.

Guilloux P. (1996), Le congé individuel de formation. Genèse et évolution d'un droit (1966-1996), Paris, L'Harmattan. 
d'Iribarne A., Lemaître A. (1987), La place des partenaires sociaux dans la formation professionnelle en France, Office des Publications officielles des Communautés européennes.

d'Iribarne A. (2001), « Trente ans de Céreq, des qualifications aux compétences : Chronique d'un oubli accepté ? ", Formation Emploi, n 76, p. 71-97.

Join-Lambert O. (2010), « La formation professionnelle : qui oriente ? qui finance ? qui contrôle ? », in Daumas J.-C. (dir.), Chatriot A., Fraboulet D., Fridenson P., Joly $\mathrm{H}$., Dictionnaire historique des patrons français, Paris, Flammarion, p. 889-892.

Luttringer J.-M. (2008), « Réflexions sur les spécificités du "paritarisme de gestion" dans le domaine de la formation professionnelle », Droit Social, $\mathrm{n}^{\circ} 12$, p. $1207-1216$.

Maggi-Germain N. (1999), « À propos de l'individualisation de la formation professionnelle continue ", Droit Social, $n^{\circ} 7 / 8$, juillet-août, p. 692-699.

Mériaux O. (1996), « Les théories de l'échange politique à l'épreuve du système français de formation professionnelle ", in Bourque R. (dir.), Les transformations des relations professionnelles, Etudes françaises et québécoises, Paris, La Documentation française, Cahier Travail et Emploi, p. 247-266.

Mériaux O. (1997), « Vers un nouveau modèle de paritarisme dans la gestion des fonds de la formation professionnelle ? ", La Revue de l'IRES, ${ }^{\circ} 24$, numéro spécial, Le paritarisme, institutions et acteurs, p. 191-208.

Mériaux O. (1999), « L'action publique partagée. Formes et dynamiques institutionnelles de la régulation politique du régime français de formation professionnelle continue », Thèse pour le doctorat en science politique, Université de Grenoble II, IEP.

Merle V. (2004), « Un accord historique ? », Droit Social, $n^{\circ}$ 5, numéro spécial, Le nouveau droit de la formation, mai, p. 455-463.

Morin M.-L. (1996), « Permanences et transformations du rôle de la négociation collective de branche », in Murray G., Morin M.-L., da Costa I. (dir.), L'état des relations professionnelles. Traditions et perspectives de recherches, Toulouse, Octarès, p. 351-375.

Nallet J.-F. (1991), « Le droit de la formation, une construction juridique fondatrice », Formation Emploi, n 34 , p. 5-13.

Pernot J.-M., Vincent C. (coord.) (2011), Les organisations patronales. Continuités et mutations des formes de représentation du patronat, Rapport IRESDARES.

Pierson P. (1995), Dismantling the Welfare State? Reagan, Thatcher and the Politics of Retrenchment, New York, Cambridge University Press.

Reynaud J.-D. (1989), Les Règles du jeu. L'action collective et la régulation sociale, Paris, Armand Colin.

Reynaud J.-D., Eyraud F., Paradeise C., Saglio J. (1990), Les systèmes de relations professionnelles, Examen critique d'une théorie, Paris, Editions du CNRS.

Reynaud J.-D. (2001), « Le management par les compétences : un essai d'analyse ", Sociologie du Travail, vol. 43, n 1, p. 7-31.

Santelman P. (2001), La formation professionnelle, nouveau droit de l'homme ?, Paris, Gallimard. 
Silvestre J.-J. (1986), « Marché du travail et crise économique : de la mobilité à la flexibilité », Formation Emploi, $n^{\circ} 14$, p. 54-62.

Tallard M. (2001), « L'introduction de la notion de compétence dans les grilles de classification. Genèse et évolution ", Sociétés contemporaines, n 41-42, p. 159-187.

Tallard M. (2004), Action publique et régulation de branche de la relation salariale, Paris, L'Harmattan.

Tanguy L. (1999), « Les chantiers de la formation permanente (1945-1971), Introduction ", Sociétés contemporaines, $n^{\circ} 35$, p. 7-18

Tanguy L. (2001), « Un mouvement social pour la formation permanente en France (1945-1970) », Education permanente, numéro spécial, La formation permanente : entre travail et citoyenneté, p. 11-28.

Verd J.-M., Vero J. (2011), « Pourquoi la flexicurité mérite-t-elle qu'on en débatte à partir de l'approche par les capacités ? ", Formation Emploi, n 113, janviermars, p. 5-14.

Verdier J.-M, Langlois P. (1972), « Aux confins de la théorie des sources du droit: une relation nouvelle entre la loi et l'accord collectif », Recueil Dalloz Sirey, $39^{\mathrm{e}}$ cahier, chron. 253.

Verdier E. (2008), « L'usage politique des idées floues, l'éducation et la formation tout au long de la vie ", in Giraud O., Warin P., Politiques publiques et démocratie, Paris, La Découverte, p. 109-135.

Vincent C. (1997), « De l'accord de juillet 1970 à la loi de 1971 : l'échec d'un paritarisme négocié dans la formation professionnelle continue ", La Revue de I'IRES, numéro spécial, Le paritarisme, institutions et acteurs, p. 153-173.

Vincent C. (2002), « Les acteurs syndicaux et patronaux face à la régionalisation de la formation professionnelle : une difficile articulation entre politiques de branches et politiques territoriales ", in Bernier C., Jobert A., Rainbird H., Saglio J., Formation, relations professionnelles et syndicalisme à l'heure de la société-monde, Paris, Québec, L'Harmattan, Les Presses de l'Université Laval, p. 41-56.

Zarifian P. (1988), « L'émergence du modèle de la compétence », in Stankiewicz F. (dir.), Les stratégies d'entreprises face aux ressources humaines, Paris, Economica, p. 77-82. 